\title{
Intraoperative head drift and eye movement: two under addressed challenges during cataract surgery
}

\author{
Kerr Brogan ${ }^{1} \cdot$ Basu Dawar ${ }^{1}$ David Lockington ${ }^{1} \cdot$ Kanna Ramaesh $^{1}$
}

Received: 11 November 2017 / Accepted: 5 January 2018 / Published online: 21 February 2018

(c) The Royal College of Ophthalmologists 2018

\begin{abstract}
Purpose To objectively measure head drift during cataract surgery, and subjectively simulate eye movements and assess impact on surgical technique.

Materials and methods Twelve consecutively recorded routine cataract operations in the Tennent Institute of Ophthalmology, Gartnavel General Hospital, Glasgow, were reviewed. The speculum was used as a fixed point and correlated with a superimposed virtual ruler to measure maximum head drift in each direction throughout the operations. To simulate intraoperative eye movement, we attached string to the cataract surgical simulator (Eyesi) eye and manually induced abduction and adduction. A calibrated scale secured to the Eyesi head ensured $5 \mathrm{~mm}$ eye movements were consistently created. Ophthalmology trainees performed the continuous curvilinear capsulorhexis (CCC) exercise without and with sequential eye movements. Movements were induced every three seconds. Scores were compared using a paired Student's $T$ test.

Results Mean head drift in the surgical recordings was $3.1 \mathrm{~mm}$ medially (range 2-7 mm), $2.9 \mathrm{~mm}$ laterally (range $2-4 \mathrm{~mm}$ ), $2.6 \mathrm{~mm}$ superiorly (range $1-5 \mathrm{~mm}$ ), and $1.9 \mathrm{~mm}$ inferiorly (range $1-4 \mathrm{~mm}$ ). In 11 of 12 cases, the operating microscope had to be adjusted for head drift. Six junior trainees completed the CCC module on the Eyesi without then with eye movements. After introducing eye movements the mean Eyesi score reduced from 92.7 to $76.9(P=0.014)$, 'roundness of rhexis' score reduced from 89.4 to $57.5(P=0.020)$, and trainees operated $17 \mathrm{~s}$ faster $(P=0.016)$.

Conclusion This study objectively demonstrates the under-reported clinical scenario of head drift during cataract surgery. By manipulating the Eyesi we have shown that eye movements reduce the quality of cataract surgery.
\end{abstract}

\section{Introduction}

Cataract surgery is the most common operation in the United Kingdom, with around 330,000 cataract operations performed each year in England alone [1]. Visual outcomes following cataract surgery are highly successful, with $94.6 \%$ of eyes with no additional ocular pathology achieving a postoperative best corrected visual acuity (BCVA) of 0.30 LogMAR or better [2]. Despite technological advances, the

Electronic supplementary material The online version of this article (https://doi.org/10.1038/s41433-018-0047-x) contains supplementary material, which is available to authorized users.

Kerr Brogan

kerrbrogan@hotmail.co.uk

1 Tennent Institute of Ophthalmology, Gartnavel General Hospital, Glasgow, Scotland surgical complication rate for posterior capsule rupture (PCR) has not been shown to decrease when comparing the Royal College of Ophthalmologists national cataract audit database results between 2011 and 2015 (1.92\% versus $1.95 \%$ respectively) $[2,3$,$] . To address this, pre-operative$ risk factor stratification tools have been created to identify challenging cases with a higher probability of PCR, and such cases can be appropriately reserved for more experienced cataract surgeons [4]. Although such tools have proved effective [5], intraoperative head and eye movements were not included in the risk analysis. From our clinical experience, we have observed that intraoperative head drift and excessive eye movements significantly increase the challenge of cataract surgery and potentially contribute to intraoperative complications. Previous studies have demonstrated the importance of simulating the management of surgical complications, with a view to achieving the best outcome [6]. The purpose of this study was twofold-to develop a method to objectively measure head drift during 


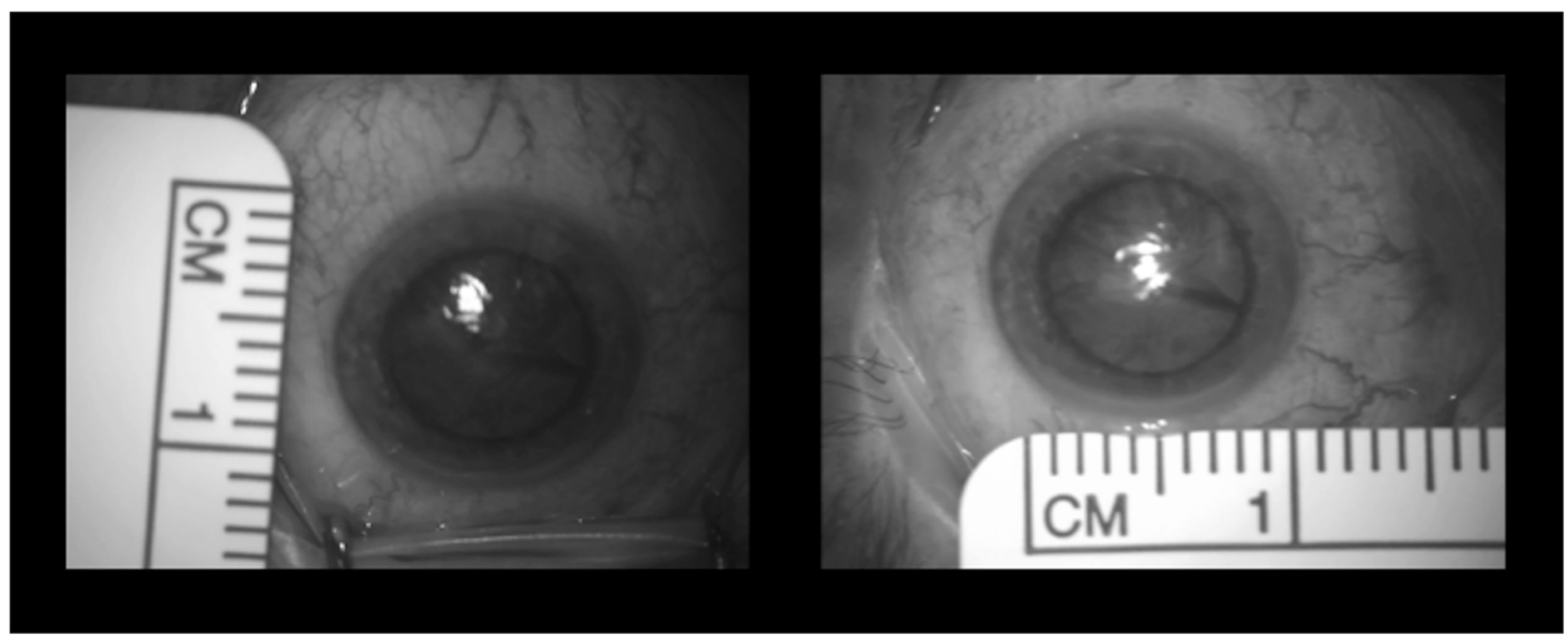

Fig. 1 Vertical and horizontal scale held up to the eye at the start of surgery

cataract surgery and secondly, to test our hypothesis that intraoperative eye movements reduce the quality of cataract surgery. For the latter, we asked ophthalmology trainees to complete the continuous curvilinear capsulorhexis (CCC) module on the ophthalmic surgical simulator (Eyesi, VR Magic, Mannheim, Germany). We observed the impact of artificially induced eye movements on their Eyesi scores.

\section{Materials and methods}

\section{Objective head drift measurements}

Twelve recorded consecutive routine cataract operations were reviewed for patient head drift and subsequent microscope repositioning. In order to objectively measure head drift, a fixed point within the recorded footage had to be identified and calibrated with a defined scale. Any landmark on the surface of the eye could be confounded by eye movements and was therefore avoided. The eyelid speculum, however, did not move secondary to eye movements and only changed position when the head drifted. The speculum from each video was therefore used as the fixed point. At the start of recording each operation a sterile ruler was held beside the eye in horizontal and vertical meridians. Corneal diameter measurements were noted. Screen shots that included the rulers were taken during video playback (Fig. 1). These images were then cropped and edited to only contain the rulers, then superimposed over the original video prior to playback (Fig. 2). Intraoperative microscope magnification adjustment by the surgeon resulted in variation in the image size during video analysis. When this occurred the superimposed scale size was re-edited to measure the same corneal diameter recorded at baseline. This ensured the video image and the superimposed scale sizes did not drift out of proportion. The eyelid speculum

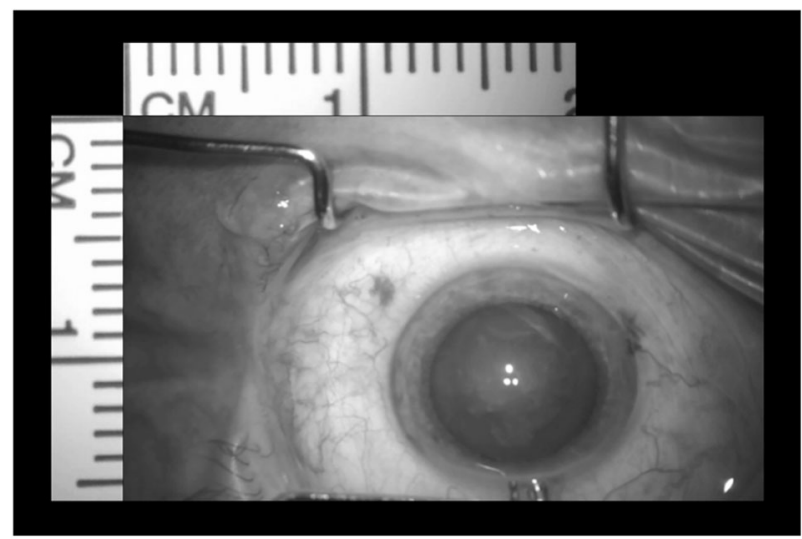

Fig. 2 Scale images cropped and superimposed over video prior to play back

was correlated with the scale during playback to measure the maximal head drift in the medial, lateral, superior, and inferior directions for all 12 surgical cases. Times when the surgeon manually repositioned the patient's head or adjusted the microscope were noted during the recordings; these movements were excluded during video analysis.

\section{Subjectively simulating eye movements}

Ophthalmology trainees (within their first 2 years of training) performed the low-tension CCC exercise from the beginner module on the Eyesi simulator (Eyesi, VR Magic, Mannheim, Germany). Each trainee completed the exercise three times. The average of these scores was used as their baseline performance. A scale measuring $5 \mathrm{~mm}$ increments was attached to the Eyesi head, and string was attached to the simulator eye and used to create abduction and adduction movements (Fig. 3). Coloured string attached was aligned with and read off the scale when moving the eye (green string on the medial scale and red string on the lateral scale on the Eyesi head). This ensured each eye movement created 


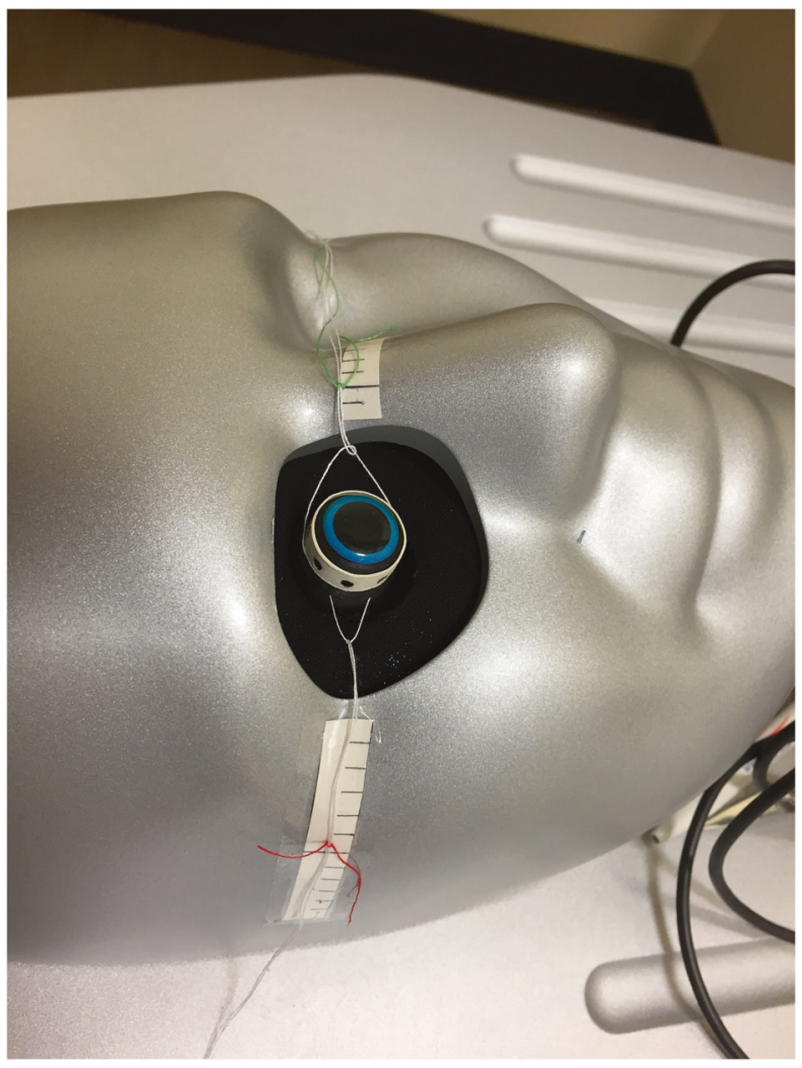

Fig. 3 Eyesi with string attached to the simulator eye and a scale to the mannequin head

measured $5 \mathrm{~mm}$. A random sequence of eye movements was generated and used for each trainee. The sequence of eye movements was recorded on an audio device, with each movement being read out at three-second intervals. This audio sequence was played back to the examiner through a pair of earphones during the exercise; so enabling the examiner to focus on reading the scale and moving the eye consistently $5 \mathrm{~mm}$ each time. The trainees then repeated the CCC exercise with the addition of these simulated eye movements (see Supplementary Online Video). Eyesi scores before and after introduction of eye movements were compared. A paired Student's $T$-test using $\mathrm{R}$ version 3.1.1 was used for statistical analysis. $P$ values of $\leq 0.05$ were considered statistically significant.

\section{Results}

\section{Objective head drift measurements}

Twelve consecutive cataract (phacoemulsification and intraocular lens implantation) surgical operations were recorded. Ten cases were performed by a consultant ophthalmologist and two by a senior ophthalmology trainee. Eight patients received topical anaesthetic, four received sub-tenons. Maximal mean head drift was greatest medially, measuring $3.1 \mathrm{~mm}$ (range $2-7 \mathrm{~mm}$ ), followed by $2.9 \mathrm{~mm}$ laterally (range $2-4 \mathrm{~mm}$ ), $2.6 \mathrm{~mm}$ superiorly (range $1-5$ $\mathrm{mm}$ ), and $1.9 \mathrm{~mm}$ inferiorly (range $1-4 \mathrm{~mm}$ ). In 11 of 12 cases, the operating microscope had to be adjusted to accommodate for head movement. No complications occurred during any of the cases.

\section{Subjectively simulating eye movements}

Six junior ophthalmologists ( 3 first year, $3 \mathrm{~s}$ year trainees) completed the eye movement simulation. In keeping with the level of inexperience, no participant had performed $>50$ complete cataract operations to date (mean number 19; range 0-41). Previous time spent to date using the Eyesi to learn various cataract-related techniques averaged $30.8 \mathrm{~h}$ (range 17-77 h). Mean Eyesi score at baseline for the CCC exercise was $92.7 \pm 4.3$. After introducing eye movements, a statistically significant deterioration in performance was found. Mean Eyesi score for the task reduced to $76.9 \pm 10.3$ $(P=0.014)$, 'roundness of CCC' score reduced from 89.4 to $57.5(P=0.02)$, and average time to complete the CCC reduced by $17 \mathrm{~s}(P=0.016$; Table 1$)$.

No complications were observed during completion of the CCC task without eye movements. With the introduction of simulated eye movements two trainees had a radial extension of CCC up to $3.3 \mathrm{~mm}$ and one trainee failed to fully complete the CCC (a small section of the rhexis flap was still attached to the anterior capsule at the end of the exercise, which went unnoticed by the trainee).

\section{Discussion}

In this study, we have attempted to objectively and subjectively address the issues of head drift and eye movements during cataract surgery. Most cataract surgeons will have experienced several challenging surgical cataract cases due to these scenarios, which can arise unpredictably, even in the absence of systemic conditions that cause uncontrolled movement disorders such as head tremor.

Intraoperative head drift causes two problems during cataract surgery: loss of centration of the operating microscope; and loss of focus. The latter is caused by the eye not only drifting to the side but also posteriorly as the patient's head turns. If the microscope is at the limit of its depth of focus when the head drifts, then the image may not be able to be fully re-focussed with the operating microscope foot pedal. Both loss of centration and focus are more problematic at higher magnification settings on the microscope due to the resulting smaller field of view and smaller depth of focus. In our study, 11 of 12 recorded surgical cases had to have manual repositioning of the patient head or microscope to compensate for head drift in order to re-gain 
Table 1 Trainees Eyesi scores before and after introduction of eye movements

\begin{tabular}{llll}
\hline & $\begin{array}{l}\text { Before movements } \\
(N \pm \text { s.d. })\end{array}$ & $\begin{array}{l}\text { After eye movements } \\
(N \pm \text { s.d. })\end{array}$ \\
\hline Eyesi score & $92.7 \pm 4.3$ & $76.9 \pm 10.3$ & $\mathbf{0 . 0 1 4}$ \\
Roundness of CCC & $89.5 \pm 11.6$ & $57.5 \pm 29.3$ & $\mathbf{0 . 0 2 0}$ \\
Time to complete exercise (seconds) & $66 \pm 19.0$ & $49 \pm 13.2$ & $0.32 \pm 0.07$ \\
Centering of CCC (mm) & $0.22 \pm 0.05$ & $0.23 \pm 0.13$ & 0.058 \\
Deviation of CCC radius from $2.2 \mathrm{~mm}$ template $(\mathrm{mm})$ & $0.18 \pm 0.07$ & 0.32 \\
\hline
\end{tabular}

Eyesi Cataract surgical simulator, $C C C$ continuous curvilinear capsulorhexis

Bold values indicate statistically significant results

optimal surgical view. We observed that the patient's head had a greater tendency to drift further in the medial direction. This is relevant because medial head drift can promote pooling of fluid in the inner canthus and subsequently submersing the corneal surface, which further deteriorates the operating view. Although our project evaluated maximal head drift (a gradual process), McCannel et al. [7] reported sudden unexpected head movements during intraocular surgery in patients receiving local anaesthetic with intravenous sedation. In particular, almost half of their patients who were snoring during surgery had sudden head movements, which increases the risk of intraoperative ocular injury [7]. In a previous closed claim analysis study, intraoperative patient movement was reported as being the second most common mechanism for ocular injury during surgery and accounted for $30 \%$ of eye injury claims against anaesthetists [8]. If sedation has to be used, evidence suggests that remifentanil sedation is associated with less head movements than is the case with propofol [9]. For certain patients, particularly those with uncontrolled tremor, there is a clear need for head stabilisation during cataract surgery. Armstrong suggested taping the patient's head in conjunction with a donut-shaped headrest to minimise head movement during cataract surgery [10]. Halladay [11] reported using a surgical headrest with a central trough in the middle to allow the surgeon to rest their hands on the edge of the headrest at the patient's eye level and minimising intraoperative hand tremor. This trough design also aimed to improve patient comfort and prevent head movement. Such devices have fallen out of fashion in ophthalmic practice as they can be seen by some as a form of 'restraint'. This was one of the reasons to perform this study. Although there is anecdotal benefit from head stabilisation, without measuring head drift before and after implementing such techniques, we are unable to quantify how effective these techniques actually are. As our method for measuring head drift is relatively straightforward, this could be easily replicated and used to investigate different methods for minimising intraoperative head drift during cataract surgery, for both the safety and better outcomes in the appropriately identified, fully consented patient.
The second part of this project was to test our hypothesis that intraoperative eye movements decrease the quality of cataract surgery. Eye movements are generally more frequent and erratic than head drift during cataract surgery and therefore more challenging to measure. For this reason we decided to create an artificial eye movement simulation using the Eyesi. We recruited trainees within the first two years of training to avoid the potential for large differences in surgical experience to confound our results. The CCC simulation was chosen because we felt this was the most realistic exercise on the Eyesi. The same sequence of eye movements was used for each trainee, the size of eye movements all measured $5 \mathrm{~mm}$, and movements were consistently generated every $3 \mathrm{~s}$. Although this was not fully representative of unpredictable eye movements experienced during real surgery, it enabled a fair comparison between different trainees. We found the Eyesi scores for the CCC exercise to be significantly poorer after introduction of eye movements, demonstrating the negative consequences on surgical performance. Interestingly, the participants were observed to operate faster after eye movements were introduced. They appeared to be under more stress due to the eye moving, and therefore sped up to complete (or get through) the exercise as quickly as possible. If trainees are unwittingly speeding up their surgery when they are under pressure then this could lead to additional complications. This concept was corroborated by two of our trainees having radial extensions of their CCC when eye movements were introduced. Several studies have shown improved performance of junior ophthalmologists in the operating room after practicing on the Eyesi [12-22]; in particular, the rates of errant CCC are significantly reduced [17, 19,]. One of the limitations of the Eyesi in preparing trainees for real cataract surgery, however, is lack of intraoperative eye movement during the exercises. Our artificial eye movement simulation gave the junior trainees an opportunity to practice the CCC on a mobile eye in a controlled and safe environment without the repercussions of complicated surgery if the CCC tore out.

In the real life setting, previous studies have attempted to grade intraoperative eye movements during cataract 
surgery. Zehetmayer et al used a scale of -5 (adverse motility) to +5 (ideal cooperation) when comparing topical versus peribulbar anaesthesia during cataract surgery; adverse motility was defined as 'ocular movement that interfered with surgery' [23]. Aslankurt et al., when grading patient cooperation during cataract surgery, used 0 (best) to 3 (worst); grade 3 was defined as 'head and/or globe movements and/or eyelid squeezing significant enough to jeopardize surgery' [24]. In addition to these studies, peribulbar and retrobulbar blocks have previously been graded in relation to akinesia achieved [25]. Although these papers illustrate the potential for utilising subjective eye movement scales in cataract surgery, no study to date has linked adverse intraoperative ocular motility to poorer surgical outcomes.

But how does all this relate to clinical practice? Our study suggests that patients with erratic eye movements should be identified as a risk factor for potential intraoperative complications, and be reserved for more experienced cataract surgeons using an akinetic form of anaesthesia. A recent Cochrane review suggested peribulbar and retrobulbar techniques to be equally as effective as each other in achieving akinesia, however, these have fallen out of common use due to the risks associated with sharp needle anaesthesia compared to the subtenon approach [25]. Control of eye movement from subtenon's anaesthetic is comparable to other anaesthetic methods [26]. Surprisingly, there are no randomised control trials comparing akinesia in subtenons with that in topical anaesthetic for cataract surgery. Intraoperative pain, on the other hand, is well established to be higher in topical anaesthetic than sub-tenons and may contribute to unwanted head and eye movements [27].

The main limitation in our study was the lack of motion tracking technology. This would have helped us track head and eye movement and calculate the velocity, distance, and rate of movement during surgery. If such technology was adapted for this use then we could potentially build a database of several cataract cases and see if there is a correlation between excessive head and eye movement with an increased risk of capsule rupture.

In conclusion, this study has started to identify and address the challenges of intraoperative head drift and eye movement during cataract surgery. We have developed a relatively straightforward and easily reproducible method for objectively measuring head drift during cataract surgery. We have also demonstrated through surgical simulation the negative impact that intraoperative eye movement can have on the quality of cataract surgery. This simulated clinical scenario could be replicated in the other units which have access to the Eyesi to enable trainee surgeons to be better prepared with regard to the impact of eye movements on their surgical techniques.

\section{Summary}

\section{What was known before}

- Head stabilisation techniques during cataract surgery have previously been proposed; however, no methods for measuring intraoperative head drift have been produced. Without measuring head movement before and after head stabilisation, we are unable to quantify how effective such techniques are.

- Most cataract surgeons will have experienced challenging surgical cataract cases due to intraoperative eye movements. Reduced quality of cataract surgery secondary to excessive ocular motility is, however, yet to be demonstrated.

\section{What this study adds}

- We have created a relatively straightforward and reproducible method for measuring head drift during cataract surgery; this may prove useful in future studies investigating head stabilisation techniques.

- By simulating eye movements on the Eyesi we have demonstrated that intraoperative eye movements can reduce the quality of cataract surgery. Our simulation could be replicated by other teaching hospitals to familiarise trainee ophthalmologists of the challenges of intraoperative eye movements in a controlled and safe environment.

Acknowledgements We thank all the junior ophthalmology trainees in Gartnavel General Hospital that took part in this study.

\section{Compliance with ethical standards}

Conflict of interest The authors declare that they have no conflict of interest.

\section{References}

1. Hospital Episode Statistics (HES). Main procedures and interventions. (accessed on 01 October 2017). http://www.hesonline.nhs. uk/Ease/servlet/ContentServer?siteID $=1937 \&$ categoryID $=537$

2. Day AC, Donachie PH, Sparrow JM, Johnston RL. Royal College of Ophthalmologists' National Ophthalmology Database. The Royal College of Ophthalmologists' National Ophthalmology Database study of cataract surgery: report 1, visual outcomes and complications. Eye (Lond). 2015;29:552-60.

3. Sparrow JM, Taylor H, Qureshi K, Smith R, Johnston RL. The cataract national data set electronic multi-centre audit of 55,567 operations: case mix adjusted surgeon's outcomes for posterior capsule rupture. Eye (Lond). 2011;25:1010-5.

4. Narendran N, Jaycock P, Johnston RL, Taylor H, Adams M, Tole DM, et al. The Cataract National Dataset electronic multicentre audit of 55,567 operations: risk stratification for posterior capsule rupture and vitreous loss. Eye (Lond). 2009;23:31-7. 
5. Addison PK, Rajendram R, Bradshaw H, Hykin PG. Using data from the Cataract National Dataset electronic multicentre audit to calculate risk of posterior capsule rupture and vitreous loss for patients on current surgical lists. Eye (Lond). 2011;25:396-7.

6. Lockington D, Belin M, McGhee CNJ. The need for all cataract surgeons to run a regular vitreous loss fire drill. Eye (Lond). 2017;31:1120-1.

7. McCannel CA, Olson EJ, Donaldson MJ, Bakri SJ, Pulido JS, Mueller D. Snoring is associated with unexpected patient head movement during monitored anesthesia care vitreoretinal surgery. Retina. 2012;32:1324-7.

8. Gild WM, Posner KL, Caplan RA, Cheney FW. Eye injuries associated with anesthesia. A closed claims analysis. Anesthesiology. 1992;76:204-8.

9. Boezaart AP, Berry RA, Nell ML, van Dyk AL. A comparison of propofol and remifentanil for sedation and limitation of movement during periretrobulbar block. J Clin Anesth. 2001;13:422-6.

10. Armstrong TA. Head immobilisation in eye surgery. Anesthesiology. 1994;81:273-4.

11. Halladay BL. A new surgical head rest. $\mathrm{Br} \mathrm{J}$ Ophthalmol. 1988;72:284-5.

12. Thomsen AS, Bach-Holm D, Kjærbo H, Højgaard-Olsen K, Subhi Y, Saleh GM, et al. Operating room performance improves after proficiency-based virtual reality cataract surgery training. Ophthalmology. 2017;124:524-31.

13. Puri S, Srikumaran D, Prescott C, Tian J, Sikder S. Assessment of resident training and preparedness for cataract surgery. J Cataract Refract Surg. 2017;43:364-8.

14. Thomsen AS, Smith P, Subhi Y, Cour M, Tang L, Saleh GM, et al. High correlation between performance on a virtual-reality simulator and real-life cataract surgery. Acta Ophthalmol. 2017;95:307-11.

15. Lam CK, Sundaraj K, Sulaiman MN, Qamarruddin FA. Virtual phacoemulsification surgical simulation using visual guidance and performance parameters as a feasible proficiency assessment tool. BMC Ophthalmol. 2016;14:88.

16. Thomsen AS, Kiilgaard JF, Kjaerbo H, la Cour M, Konge L. Simulation-based certification for cataract surgery. Acta Ophthalmol. 2015;93:416-21.
17. Daly MK, Gonzalez E, Siracuse-Lee D, Legutko PA. Efficacy of surgical simulator training versus traditional wet-lab training on operating room performance of ophthalmology residents during the capsulorhexis in cataract surgery. J Cataract Refract Surg. 2013;39:1734-41.

18. Spiteri AV, Aggarwal R, Kersey TL, Sira M, Benjamin L, Darzi AW, et al. Development of a virtual reality training curriculum for phacoemulsification surgery. Eye (Lond). 2014;28:78-84.

19. McCannel CA, Reed DC, Goldman DR. Ophthalmic surgery simulator training improves resident performance of capsulorhexis in the operating room. Ophthalmology. 2013;120:2456-61.

20. Saleh GM, Lamparter J, Sullivan PM, O'Sullivan F, Hussain B, Athanasiadis I, et al. The international forum of ophthalmic simulation: developing a virtual reality training curriculum for ophthalmology. Br J Ophthalmol. 2013;97:789-92.

21. Naseri A, Chang DF. Assessing the value of simulator training on residency performance. J Cataract Refract Surg. 2012;38:188-9. author reply 189

22. Belyea DA, Brown SE, Rajjoub LZ. Influence of surgery simulator training on ophthalmology resident phacoemulsification performance. J Cataract Refract Surg. 2011;37:1756-61.

23. Zehetmayer M, Radax U, Ch Skorpik, Menapace R, Schemper M, Weghaupt $\mathrm{H}$, et al. Topical versus peribulbar anaesthesia in clear cornea cataract surgery. J Cataract Refract Surg. 1996;22:480-84.

24. Aslankurt M, Aslan L, Baskan AM, Aksoy A, Silav E, Yildiz H. Pain and cooperation in patients having dominant or non dominant side phacoemulsification. J Cataract Refract Surg. 2014;40:199-202.

25. Alhassan MB, Kyari F, Ejere HO. Peribulbar versus retrobulbar anaesthesia for cataract surgery. Cochrane Database Syst Rev. 2015;2:CD004083.

26. Guise PA. Sub-Tenon anesthesia: a prospective study of 6000 blocks. Anesthesiology. 2003;98:964-8.

27. Guay J, Sales K. Sub-Tenon's anaesthesia versus topical anaesthesia for cataract surgery. Cochrane Database Syst Rev. 2015;27: CD006291. 